\title{
Numerical Solution of Laplace Equation using Fuzzy Data
}

\author{
Raphel Kumar Saikia \\ Associate Professor \\ Jorhat Institute of Science and Technology \\ Jorhat-785010, Assam, INDIA
}

\begin{abstract}
In this paper, we have discussed fuzzification of elliptic partial differential equation taking Laplace Equation in two variable into consideration. While solving this equation numerically at different grid points, on the other hand we want to observe findings using fuzzy intervals. Finite difference method is applied in solving the equation numerically.
\end{abstract}

\section{Keywords}

$\alpha$-cut, fuzzy membership function(f.m.f.),interval of confidence, triangular fuzzy number(t.f.n.).

\section{INTRODUCTION}

The concept of Fuzzy differential equation was first introduced by Chang and Zadeh [1]. Dubois and Prade [2] has given the extension principle. In this paper, we first applied finite difference method to solve Laplace equation numerically, then fuzzified.

\section{BASIC CONCEPT AND DEFINITIONS}

A triangular Fuzzy number $\mu$ is defined by three real numbers with base as the interval $[a, c]$ and $b$ as the vertex of the triangle.The membership functions are defined as follows:

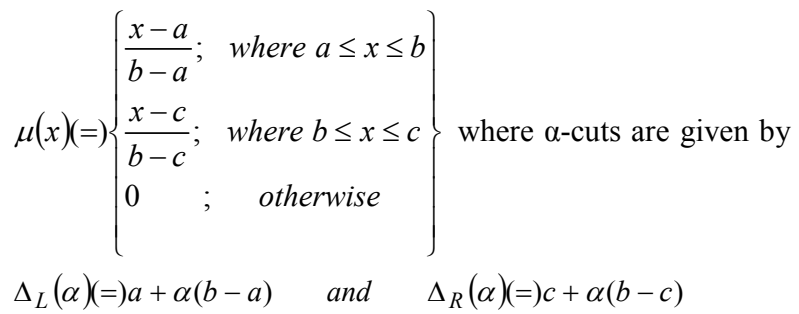

\subsection{Application of Finite Difference} Method in Laplace Equation

The Laplace's Equation in two variable is

$\frac{\partial^{2} u}{\partial^{2} x}+\frac{\partial^{2} u}{\partial^{2} y}=0 \Rightarrow u_{x x}+u_{y y}=0-----------(1)$

which is very often encountered in heat and mass transfer theory, fluid mechanics, elasticity, electrostatics, and other areas of mechanics and physics, is an elliptic partial differential equation since $\mathrm{B}^{2}-4 \mathrm{AC}=-4<0$.
Replacing $\mathrm{u}_{\mathrm{xx}}$ and $\mathrm{u}_{\mathrm{yy}}$ by finite difference method, (1) becomes

$$
\begin{aligned}
& \frac{u_{i-1, j}-2 u_{i, j}+u_{i+1, j}}{h^{2}}+\frac{u_{i, j-1}-2 u_{i, j}+u_{i, j+1}}{k^{2}}=0 \\
& \Rightarrow u_{i, j}=\frac{1}{4}\left[u_{i-1, j}+u_{i+1, j}+u_{i, j-1}+u_{i, j+1}\right]--------(2)
\end{aligned}
$$

[Taking meshes to be of square nature]

i.e. the value of $u$ at any mesh point is the arithmetic mean of its values at the four neighboring mesh points to the left, right, above and below which is called Standard Five Point Formula (SFPF) could be represented as per Fig-1. Here we will use two formulae called Standard Five Point Formula (SFPF) and Diagonal Five Point Formula (DFPF) represented respectively by diagrams Fig-1 and Fig-2.

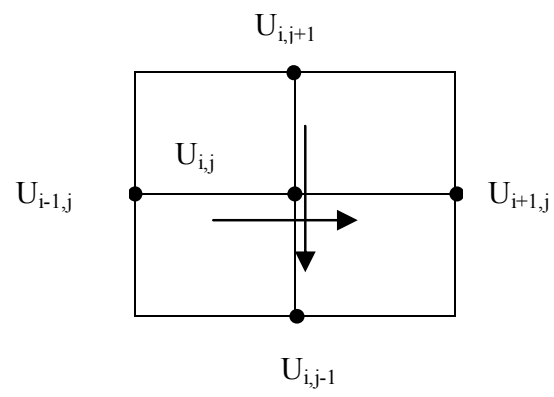

Fig-1

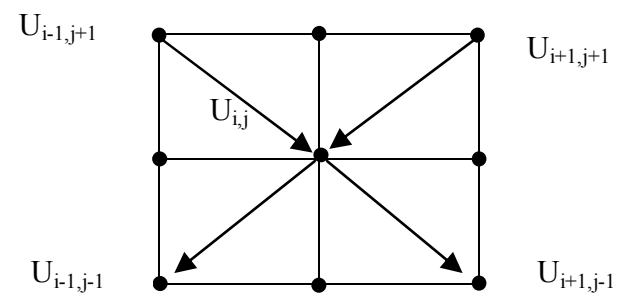

Fig-2

We will use Diagonal Five Point Formula (DFPF) wherever necessary as

$u_{i, j}=\frac{1}{4}\left[u_{i+1, j+1}+u_{i-1, j-1}+u_{i-1, j+1}+u_{i+1, j-1}\right]$ 


\section{APPLICATION OF FUZZY}

\section{INTERVAL IN LAPLACE EQUATION}

Boundary points have been taken from $\mathrm{C}_{1}$ to $\mathrm{C}_{16}$ due to equation no. (1) with fuzzy intervals as given in Table-1.

\begin{tabular}{|l|l|}
\hline $\mathrm{C}_{1}(=)\left[l_{1,1} ; l_{1,2} ; l_{1,3}\right]$ & $\mathrm{C}_{2}(=)\left[l_{2,1 ;} l_{2,2 ;} l_{2,3}\right]$ \\
\hline $\mathrm{C}_{3}(=)\left[l_{3,1 ;} l_{3,2 ;} l_{3,3}\right]$ & $\mathrm{C}_{4}(=)\left[l_{4,1 ;} l_{4,2 ;} l_{4,3}\right]$ \\
\hline $\mathrm{C}_{5}(=)\left[l_{5,1} l_{5,2 ;} l_{5,3}\right]$ & $\mathrm{C}_{6}(=)\left[l_{6,1 ;} l_{6,2 ;} l_{6,3}\right]$ \\
\hline $\mathrm{C}_{7}(=)\left[l_{7,1 ;} l_{7,2 ;} l_{7,3}\right]$ & $\mathrm{C}_{8}(=)\left[l_{8,1 ;} l_{8,2 ;} l_{8,3}\right]$ \\
\hline $\mathrm{C}_{9}(=)\left[l_{9,1 ;} l_{9,2 ;} l_{9,3}\right]$ & $\mathrm{C}_{10}(=)\left[l_{10,1 ;} l_{10,2 ;} l_{10,3}\right]$ \\
\hline $\mathrm{C}_{11}(=)\left[l_{11,1} ; l_{11,2 ;} l_{11,3}\right]$ & $\mathrm{C}_{12}(=)\left[l_{12,1 ;} l_{12,2 ;} l_{12,3}\right]$ \\
\hline $\mathrm{C}_{13}(=)\left[l_{13,1 ;} l_{13,2 ;} l_{13,3}\right]$ & $\mathrm{C}_{14}(=)\left[l_{14,1 ;} l_{14,2 ;} l_{14,3}\right]$ \\
\hline $\mathrm{C}_{15}(=)\left[l_{15,1 ;} l_{15,2 ;} l_{15,3}\right]$ & $\mathrm{C}_{16}(=)\left[l_{16,1 ;} l_{16,2 ;} l_{16,3}\right]$ \\
\hline
\end{tabular}

\section{Table-1}

Moreover $\mathrm{u}_{1}$ to $\mathrm{u}_{9}$ are nine interior points due to the square grids as shown in the Fig-3.

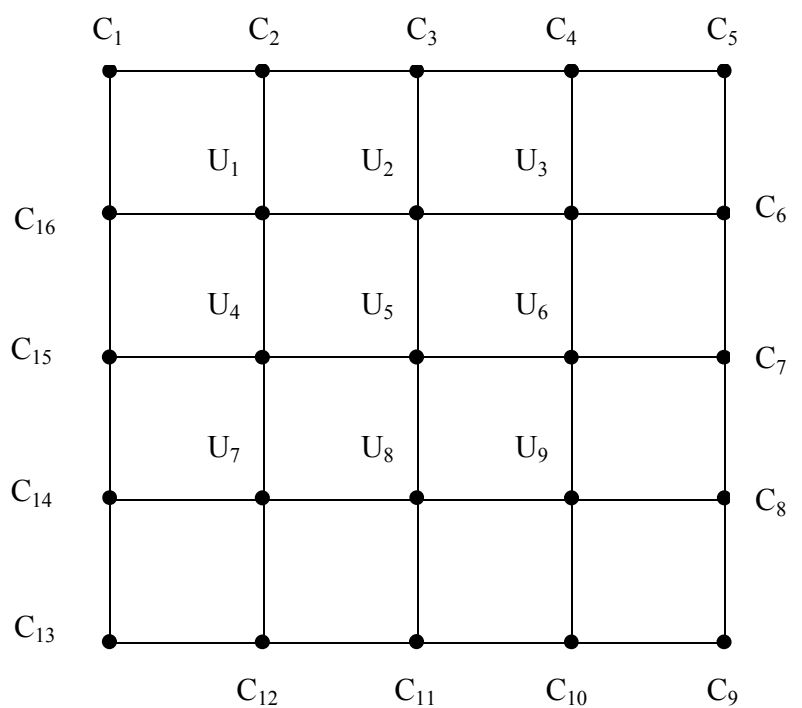

Fig-3

Now to evaluate initial value of $\mathrm{u}_{5}$ i.e. $u_{5}^{(0)}$ using Standard Five Point Formula as

$u_{5}^{(0)}\left(\Rightarrow\left[\frac{l_{3,1}+l_{11,1}+l_{15,1}+l_{7,1}}{4}, \frac{l_{3,2}+l_{11,2}+l_{15,2}+l_{7,2}}{4}, \frac{l_{3,3}+l_{11,3}+l_{15,3}+l_{7,3}}{4}\right]\right.$
Fuzzy membership functions (f.m.f.) and respective $\alpha$-cuts of $\mathrm{C}_{3}, \mathrm{C}_{11}, \mathrm{C}_{15}$ and $\mathrm{C}_{7}$ are respectively

$$
\mu_{C_{3}}(x)(=)\left\{\begin{array}{ll}
\frac{x-l_{3,1}}{l_{3,2}-l_{3,1}} ; & l_{3,1} \leq x \leq l_{3,2} \\
\frac{-x+l_{3,3}}{l_{3,3}-l_{3,2}} ; & l_{3,2} \leq x \leq l_{3,3} \\
0 \quad & \text { Otherwise }
\end{array}\right\}
$$

Hence $\alpha$-cut of $\mathrm{C}_{3}$ is

$\left[C_{3}\right]^{(\alpha)}(=)\left[\left(l_{3,2}-l_{3,1}\right) \alpha+l_{3,1},-\left(l_{3,3}-l_{3,2}\right) \alpha+l_{3,3}\right]$

$\mu_{C_{11}}(x)\left(=\left\{\begin{array}{ll}\frac{x-l_{11,1}}{l_{11,2}-l_{11,1}} ; & l_{11,1} \leq x \leq l_{11,2} \\ \frac{-x+l_{11,3}}{l_{11,3}-l_{11,2}} ; & l_{11,2} \leq x \leq l_{11,3} \\ 0 \quad ; \text { Otherwise }\end{array}\right\}\right.$

Hence $\alpha$-cut of $\mathrm{C}_{11}$ is

$\left[C_{11}\right]^{(\alpha)}(=)\left[\left(l_{11,2}-l_{11,1}\right) \alpha+l_{11,1},-\left(l_{11,3}-l_{11,2}\right) \alpha+l_{11,3}\right]$

$\mu_{C_{15}}(x)(=)\left\{\begin{aligned} \frac{x-l_{15,1}}{l_{15,2}-l_{15,1}} ; & l_{15,1} \leq x \leq l_{15,2} \\ \frac{-x+l_{15,3}}{l_{15,3}-l_{15,2}} ; & l_{15,2} \leq x \leq l_{15,3} \\ 0 \quad ; & \text { Otherwise }\end{aligned}\right\}$

Hence $\alpha$-cut of $\mathrm{C}_{15}$ is

$\left[C_{15}\right]^{(\alpha)}(=)\left[\left(l_{15,2}-l_{15,1}\right) \alpha+l_{15,1},-\left(l_{15,3}-l_{15,2}\right) \alpha+l_{15,3}\right]$

$\mu_{C_{7}}(x)(=)\left\{\begin{array}{ll}\frac{x-l_{7,1}}{l_{7,2}-l_{7,1}} ; & l_{7,1} \leq x \leq l_{7,2} \\ \frac{-x+l_{7,3}}{l_{7,3}-l_{7,2}} ; & l_{7,2} \leq x \leq l_{7,3} \\ 0 \quad ; & \text { Otherwise }\end{array}\right\}$

Hence $\alpha$-cut of $\mathrm{C}_{7}$ is

$$
\left[C_{7}\right]^{(\alpha)}(=)\left[\left(l_{7,2}-l_{7,1}\right) \alpha+l_{7,1},-\left(l_{7,3}-l_{7,2}\right) \alpha+l_{7,3}\right]
$$

Hence from (4), interval of confidence for $u_{5}^{(0)}$ is

$\left[u_{5}^{(0)}\right]^{(\alpha)} \Leftrightarrow\left[\begin{array}{l}\frac{\left(l_{3,2}-l_{3,1}\right)+\left(l_{1,2}-l_{1,1}\right)+\left(l_{152}-l_{151}\right)+\left(l_{7,2}-l_{7,1}\right)}{4} \alpha+\frac{l_{3,1}+l_{111}+l_{151}+l_{7,1}}{4}, \\ \frac{-\left(l_{3,3}-l_{3,2}\right)-\left(l_{1,3}-l_{1,2}\right)-\left(l_{153}-l_{1,2}\right)-\left(l_{7,3}-l_{7,2}\right)}{4} \alpha+\frac{l_{3,3}+l_{1,3}+l_{153}+l_{7,3}}{4}\end{array}\right]$ 
To retain two roots with $\alpha \varepsilon[0,1]$, let

$$
\begin{aligned}
& x_{1}=\frac{\left(l_{3,2}-l_{3,1}\right)+\left(l_{1,2}-l_{1,1}\right)+\left(l_{152}-l_{151}\right)+\left(l_{7,2}-l_{7,1}\right)}{4} \alpha+\frac{l_{3,1}+l_{1,1}+l_{151}+l_{7,1}}{4} \\
& x_{2}=\frac{-\left(l_{3,3}-l_{3,2}\right)-\left(l_{1,3}-l_{1,2}\right)-\left(l_{153}-l_{152}\right)-\left(l_{7,3}-l_{7,2}\right)}{4} \alpha+\frac{l_{3,3}+l_{1,3}+l_{153}+l_{7,3}}{4}
\end{aligned}
$$

Hence $\alpha=\frac{4 x_{1}-\left(l_{3,1}+l_{11,1}+l_{15,1}+l_{7,1}\right)}{\left(l_{3,2}+l_{11,2}+l_{15,2}+l_{7,2}\right)-\left(l_{3,1}+l_{11,1}+l_{15,1}+l_{7,1}\right)}$

And $\alpha=\frac{-4 x_{2}+\left(l_{3,3}+l_{11,3}+l_{15,3}+l_{7,3}\right)}{\left(l_{3,3}+l_{11,3}+l_{15,3}+l_{7,3}\right)-\left(l_{3,2}+l_{11,2}+l_{15,2}+l_{7,2}\right)}$

Hence f.m.f. for $u_{5}^{(0)}$ is

$$
\mu_{u_{5}^{(0)}}(x)=\left\{\begin{array}{c}
\frac{4 x-\left(l_{3,1}+l_{11,1}+l_{15,1}+l_{7,1}\right)}{\left(l_{3,2}-l_{3,1}\right)+\left(l_{1,2}-l_{1,1}\right)+\left(l_{15,2}-l_{15,1}\right)+\left(l_{7,2}-l_{7,1}\right)} \\
\text { where } \frac{1}{4}\left(l_{3,1}+l_{1,1}+l_{15,1}+l_{7,1}\right) \leq x \leq \frac{1}{4}\left(l_{3,2}+l_{11,2}+l_{15,2}+l_{7,2}\right) \\
\frac{-4 x+\left(l_{3,3}+l_{113}+l_{15,3}+l_{7,3}\right)}{\left(l_{3,3}-l_{3,2}\right)+\left(l_{1,3}-l_{1,2}\right)+\left(l_{15,3}-l_{15,2}\right)+\left(l_{7,3}-l_{7,2}\right)} \\
\text { where } \frac{1}{4}\left(l_{3,2}+l_{1,2}+l_{15,2}+l_{7,2}\right) \leq x \leq \frac{1}{4}\left(l_{3,3}+l_{113}+l_{15,3}+l_{7,3}\right) \\
0 \quad \text { Otherwise }
\end{array}\right\}
$$

(9)

In a similar way we can find out fuzzy membership functions for

$u_{1}^{(0)}, u_{3}^{(0)}, u_{9}^{(0)}, u_{7}^{(0)}, u_{2}^{(0)}, u_{6}^{(0)}, u_{8}^{(0)}$ and $u_{4}^{(0)}$.

Next successive approximations with their f.m.f. as required may be obtained from previous approximations and specified boundary conditios.

\section{A NUMERICAL EXAMPLE}

Let us consider the Laplace Equation in two variables

$$
\begin{aligned}
& U_{x x}(+) U_{y y}(=) 0 \text { - } \\
& (10) \\
& \text { in the region } \mathrm{R}(=)\{(x, y): 0 \leq x \leq 4,0 \leq y \leq 4\} \text { with } \\
& \text { boundary conditions } u(0, y)(=) 0, u(x, 0)(=) \frac{x^{2}}{2}, u(x, y)(=) x^{2}, \\
& \mathrm{u}(x, y)(=) 8(+) 2 y \text { Leibmann's iteration process will be }
\end{aligned}
$$
applied to solve eqn.(10) which is

$u_{i, j}^{(n+1)}(=) \frac{1}{4}\left[u_{i-1, j}^{(n+1)}(+) u_{i+1, j}^{(n)}(+) u_{i, j-1}^{(n)}(+) u_{i, j+1}^{(n+1)}\right]$

Initial values of the $u_{i}$ 's may be calculated with the help of Standard Five Point formula (SFPF) and Diagonal Five Point Formula (DFPF).
For given boundary conditions, the boundary points and their respective numerical values are

$\mathrm{C}_{1}=0, \mathrm{C}_{2}=1, \mathrm{C}_{3}=4, \mathrm{C}_{4}=9, \mathrm{C}_{5}=16, \mathrm{C}_{6}=14, \mathrm{C}_{7}=12, \mathrm{C}_{8}=10$ $, \mathrm{C}_{9}=8, \mathrm{C}_{10}=4.5, \mathrm{C}_{11}=2, \mathrm{C}_{12}=0.5, \mathrm{C}_{13}=0, \mathrm{C}_{14}=0, \mathrm{C}_{15}=0$ , $\mathrm{C}_{16}=0$.

Now to evaluate initial values $u_{i}^{\prime} s \quad(i=1,2, \ldots \ldots, 9)$

$$
u_{5}^{(0)}(=) \frac{1}{4}\left[C_{3}(+) C_{11}(+) C_{7}(+) C_{15}\right]
$$$$
\Rightarrow u_{5}^{(0)}(=)[4.449,4.5,4.501]
$$

Now to find f.m.f. and respective interval of confidence of these four $C_{i}^{\prime} s$ as

$$
\mu_{C_{3}}(x)\left(=\left\{\begin{array}{lr}
\frac{x-3.999}{4-3.999} ; & 3.999 \leq x \leq 4 \\
\frac{-x+4}{4.001-4} ; & 4 \leq x \leq 4.001 \\
0 \quad ; & \text { otherwise }
\end{array}\right.\right.
$$
$C_{3}^{(\alpha)}(=)[.001 \alpha+3.999,-.001 \alpha+4.001]$

$$
\mu_{C_{11}}(x)\left(=\left\{\begin{array}{lc}
\frac{x-1.999}{2-1.999} ; & 1.999 \leq x \leq 2 \\
\frac{-x+2.001}{2.001-2} ; & 2 \leq x \leq 2.001 \\
0 \quad ; & \text { otherwise }
\end{array}\right.\right.
$$

Interval of confidence for $C_{11}$ is $C_{11}^{(\alpha)}(=)[.001 \alpha+1.999,-.001 \alpha+2.001]$

$$
\mu_{C_{7}}(x)(=)\left\{\begin{array}{cl}
\frac{x-11.999}{12-11.999} ; & 11.999 \leq x \leq 12 \\
\frac{-x+12.001}{12.001-12} ; & 12 \leq x \leq 12.001 \\
0 \quad ; & \text { otherwise }
\end{array}\right.
$$

Interval of confidence for $C_{7}$ is $C_{7}^{(\alpha)}(=)[.001 \alpha+11.999,-.001 \alpha+12.001]$

And

$$
\mu_{C_{15}}(x)(=)\left\{\begin{array}{lc}
\frac{x+.001}{0+.001} ; & -0.001 \leq x \leq 0 \\
\frac{-x+.001}{0.001-0} ; & 0 \leq x \leq 0.001 \\
0 \quad ; & \text { otherwise }
\end{array}\right.
$$

Interval of confidence for $C_{15}$ is $C_{15}^{(\alpha)}(=)[0.001 \alpha-0.001,-0.001 \alpha+0.001]$

Hence interval of confidence for $u_{5}^{(0)}$ is

$$
\left\lfloor\left. u_{5}^{(0)}\right|^{(\alpha)}(=)[0.001 \alpha+4.499,-0.001 \alpha+4.501]\right.
$$

We are to retain two roots with $\alpha \in[0,1]$. 
Let $0.001 \alpha+4.499=x_{1}$ and $-0.001 \alpha+4.501=x_{2}($ say $)$ $\Rightarrow \alpha=\frac{x_{1}-4.499}{0.001} \quad$ and $\quad \alpha=\frac{-x_{2}+4.501}{0.001}$

Hence f. m. f. for $u_{5}^{(0)}$ is

$$
\mu_{u_{5}^{(0)}}(x)(=) \begin{cases}\frac{x-4.499}{4.5-4.499} ; & 4.499 \leq x \leq 4.5 \\ \frac{-x+4.501}{4.501-4.5} ; & 4.5 \leq x \leq 4.501------(13) \\ 0 \quad ; & \text { Otherwise }\end{cases}
$$

Similarly f.m.f. for $u_{1}^{(0)}, u_{3}^{(0)}, u_{9}^{(0)}, u_{7}^{(0)}, u_{2}^{(0)}, u_{6}^{(0)}, u_{8}^{(0)} \&$ $u_{4}^{(0)}$ are respectively

$$
\mu_{u_{1}^{(0)}}(x)\left(=\left\{\begin{array}{cc}
\frac{x-2.124}{2.125-2.124} ; & 2.124 \leq x \leq 2.125 \\
\frac{-x+2.126}{2.126-2.125} ; & 2.125 \leq x \leq 2.126 \\
0 \quad ; & \text { otherwise }
\end{array}\right.\right.
$$$$
\mu_{u_{3}^{(0)}}(x)(=)\left\{\begin{array}{rr}
\frac{x-9.124}{9.125-9.124} ; & 9.124 \leq x \leq 9.125 \\
\frac{-x+9.126}{9.126-9.125} ; & 9.125 \leq x \leq 9.126 \\
0 \quad ; & \text { otherwise }
\end{array}\right.
$$$$
\mu_{u_{9}^{(0)}}(x)(=) \begin{cases}\frac{x-6.624}{6.625-6.624} ; & 6.624 \leq x \leq 6.625 \\ \frac{-x+6.626}{6.626-6.625} ; & 6.625 \leq x \leq 6.626\end{cases}
$$

$0 \quad ; \quad$ otherwise

$\mu_{u_{7}^{(0)}}(x)(=) \begin{cases}\frac{x-1.624}{1.625-1.624} ; & 1.624 \leq x \leq 1.625 \\ \frac{-x+1.626}{1.626-1.625} ; & 1.625 \leq x \leq 1.626 \\ 0 \quad ; & \text { otherwise }\end{cases}$

$\mu_{u_{2}^{(0)}}(x)(=) \begin{cases}\frac{x-4.9365}{4.9375-4.9365} ; & 4.9365 \leq x \leq 4.9375 \\ \frac{-x+4.9385}{4.9385-4.9375} ; & 4.9375 \leq x \leq 4.9385\end{cases}$

otherwise

$\mu_{u_{6}^{(0)}}(x)(=) \begin{cases}\frac{x-8.0615}{8.0625-8.0615} ; & 8.0615 \leq x \leq 8.0625 \\ \frac{-x+8.0635}{8.0635-8.0625} ; & 8.0625 \leq x \leq 8.0635\end{cases}$

otherwise $\mu_{u_{8}^{(0)}}(x)\left(=\left\{\begin{array}{cr}\frac{x-3.6865}{3.6875-3.6865} ; & 3.6865 \leq x \leq 3.6875 \\ \frac{-x+3.6885}{3.6885-3.6875} ; & 3.6875 \leq x \leq 3.6885 \\ 0 \quad ; & \text { otherwise }\end{array}\right.\right.$

$\mu_{u_{4}^{(0)}}(x)\left(=\left\{\begin{array}{cc}\frac{x-2.0615}{2.0625-2.0615} ; & 2.0615 \leq x \leq 2.0625 \\ \frac{-x+2.0635}{2.0635-2.0625} ; & 2.0625 \leq x \leq 2.0635 \\ 0 \quad ; & \text { otherwise }\end{array}\right.\right.$

Similarly f.m.f.of fourth approximations have been found as

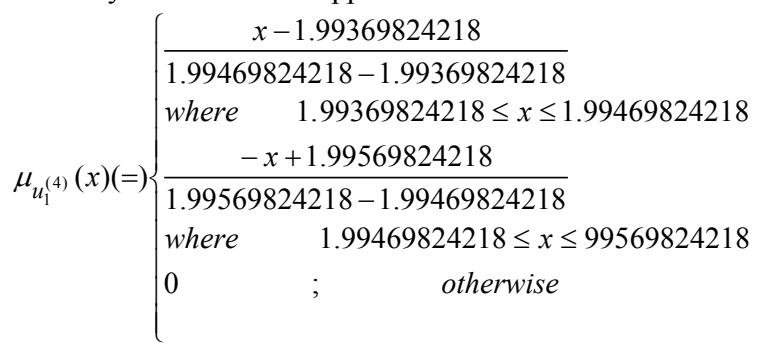

$\mu_{u_{2}^{(4)}}(x)(=)\left\{\begin{array}{l}\frac{x-4.91832202145}{4.91932202145-4.91832202145} \\ \text { where } \quad 4.91832202145 \leq x \leq 4.91932202145 \\ \frac{-x+4.92032202145}{4.92032202145-4.91932202145} \\ \text { where } \quad 4.91932202145 \leq x \leq 4.92032202145 \\ 0 \quad ; \quad \text { otherwise }\end{array}\right.$

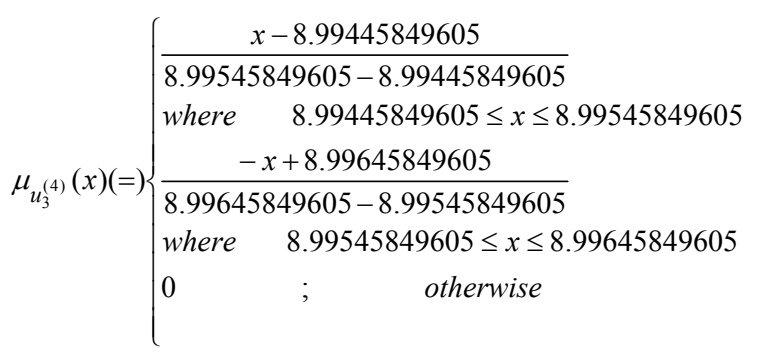

$\mu_{u_{4}^{(4)}}(x)(=)\left\{\begin{array}{l}\frac{x-2.06114428709}{2.06214428709-2.06114428709} \\ \text { where } 2.06114428709 \leq x \leq 2.06214428709 \\ \frac{-x+2.06314428709}{2.06314428709-2.06214428709} \\ \text { where } \quad 2.06214428709 \leq x \leq 2.06314428709 \\ 0 \quad ; \quad \text { otherwise }\end{array}\right.$ 


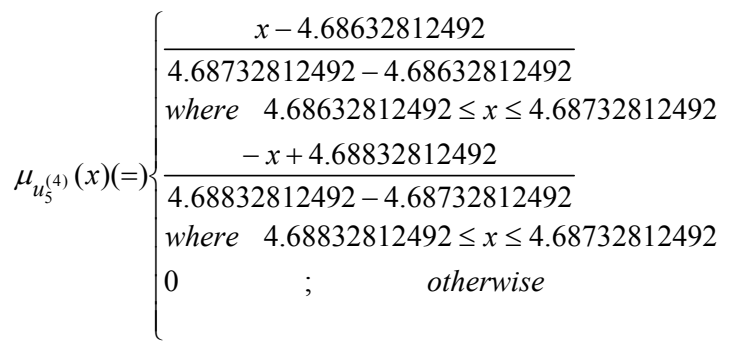

$\mu_{u_{6}^{(4)}}(x)(=)\left\{\begin{array}{l}\frac{x-8.06143704217}{8.06243704217-8.06143704217} \\ \text { where } 8.06143704217 \leq x \leq 8.06243704217 \\ \frac{-x+8.06343704217}{8.06343704217-8.06243704217} \\ \text { where } 8.06243704217 \leq x \leq 8.06343704217 \\ 0 \quad ; \quad \text { otherwise }\end{array}\right.$

$\mu_{u_{7}^{(4)}}(x)(=)\left\{\begin{array}{l}\frac{x-1.56586962889}{1.56686962889-1.56586962889} \\ \text { where } 1.56586962889 \leq x \leq 1.56686962889 \\ \frac{-x+1.56786962889}{1.56786962889-1.56686962889} \\ \text { where } 1.56686962889 \leq x \leq 1.56786962889 \\ 0 \quad ; \quad \text { otherwise }\end{array}\right.$

$\mu_{u_{8}^{(4)}}(x)(=)\left\{\begin{array}{l}\frac{x-3.70424685662}{3.70524685662-3.70424685662} \\ \text { where } 3.70424685662 \leq x \leq 3.70524685662 \\ \frac{-x+3.70624685662}{3.70624685662-3.70524685662} \\ \text { where } 3.70524685662 \leq x \leq 3.70624685662 \\ 0 \quad ; \quad \text { otherwise }\end{array}\right.$

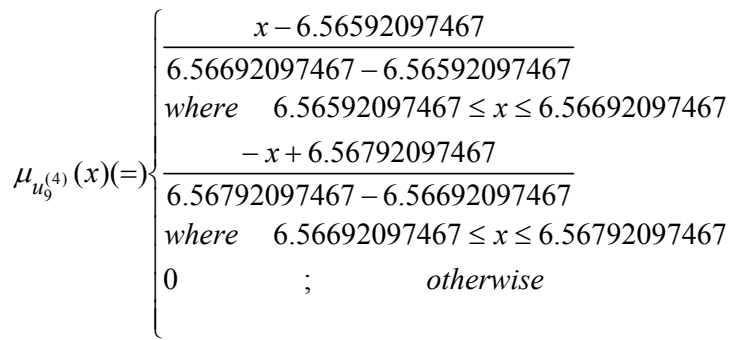

\section{CONCLUSION}

Here we have given f.m.f. for initial approximations with fourth approximations in solving the numerical example. This may however be increased up to desired accuracy.

\section{REFERENCES}

[1] Chang, S.L.,Zadeh,L.A.1972. On Fuzzy mapping and control, IEEE Trans. Systems man cyber net,2(1972) 3034.

[2] Dubois,D.,Prade,H.1982. Towards Fuzzy differential calculus, Fuzzy sets and systems, Part 3,8(1982) 225233.

[3] Grewal, B.S. 2010. Numerical methods in Engineering and sciences with Programs in $\mathrm{C} \& \mathrm{C}^{++}$, Khanna Publishers, New Delhi-110002 pp. 343-348.

[4] Baruah, Hemanta K. 1999. Set Superimposition and its applications to the Theory of Fuzzy Sets, Journal of Assam Science Society, Vol. 40, Nos. 1 \& 2, 25-31.

[5] Baruah, Hemanta K. 2010a. Construction of the Membership Function of a Fuzzy Number, ICIC Express Letters.

[6] Baruah, Hemanta K. 2010b. The Mathematics of Fuzziness: Myths and Realities, Lambert Academic Publishing, Saarbruken, Germany.

[7] Kaufmann, A., and Gupta, M. M. (1984). Introduction to Fuzzy Arithmetic, Theory and Applications, Van Nostrand Reinhold Co. Inc., Wokingham, Berkshire.

[8] Zadeh, L.A.(1968). Probability Measure of Fuzzy Events, Journal of Mathematical Analysis and Applications, Vol. 23 No. 2, August 1968 (pp 421-427).

[9] Sastry,S.S.(2009). Introductory Methods of Numerical Analysis, PHI Learning Private Limited, New Delhi110001 . 